\title{
Misperceptions of comprehension difficulties of stroke patients by doctors, nurses and relatives
}

\author{
Roshan McClenahan, Marie Johnston, Yvonne Densham
}

\begin{abstract}
Doctors, nurses and relatives involved with 30 recently aphasic stroke patients were asked to predict how the patient would perform on a comprehension test. Results show that not only do doctors, nurses and relatives underestimate the receptive disability of these patients, but they also illustrate a lack of agreement between health professionals. Implications for management are considered.
\end{abstract}

Aphasia as a result of cerebrovascular accident (CVA) is a common impairment, affecting about $30 \%$ of immediate survivors. Wade et al estimate that 60 such patients will be referred for speech therapy each year in an average United Kingdom Health District of 250000 people. $^{1}$

There is evidence that nurses and therapists caring for CVA patients do not agree about communication, mobility, self-care, pain and incontinence problems ${ }^{2}$ but information is lacking on their assessments of aphasia. Three studies have shown that relatives underestimate the impairments of aphasic patients ${ }^{34}$ even when only estimates made with high confidence are considered. ${ }^{5}$ If the health professionals are equally inaccurate in their assessment of aphasia, then misdiagnosis may occur and the management of these patients may be poorer as a result.

The aims of this study were first, to assess the accuracy of doctors, nurses and relatives in estimating the impairments of objectively assessed aphasic patients and second, to examine the extent of their agreement.

\section{Subjects and methods \\ Subjects}

The study sample was a series of 30 hospital inpatients referred to the speech therapy department as aphasic following a recent first CVA. The selection criteria excluded two patients with previous communication problems, 15 patients for whom English was not their primary language, three with psychiatric problems and one with a significant auditory/visual deficit. Six were excluded because they did not have a relative (or carer) who knew them well premorbidly and visited them in hospital at least three times each week. In addition, one refused permission and 16 were unavailable for testing (deceased, discharged to another hospital, coma). Two patients were excluded as incomplete data had been collected.

The patients, 17 men and 13 women, had an average (SD) age of $71.2(11.0)$ years. They were tested three to 24 days post CVA (mean $=12$ days). Twenty four had hemiplegia and six hemianopia. Medical notes for one patient were not available.

The relatives were: five wives, four husbands, 16 other relatives and five other carers. Twenty two house officers and 26 State Registered nurses were directly responsible for the patients' recent care.

\section{Aphasia tests}

The Auditory-Verbal Comprehension subtest of the Western Aphasia Battery (WAB) ${ }^{67}$ was used in this study. It has three sections: Yes/ No questions, such as: "Are the lights on in this room?"; Word Discrimination tasks, such as: "Point to the comb" and Sequential Commands, such as: "Point to the window and then the door".

\section{Procedure}

For each patient, the respondents (doctor, nurse, relative) were presented with the test materials and given written instructions asking them to predict the test result for each item of the WAB. At the same time, a speech therapist tested each patient on the same test, giving an objective measure of the performance. These four assessments on each patient were completed within 24 hours (except for one case which took 48 hours). Assessments were made independently and results were not shared between relatives or professionals. The majority of professionals completed only one assessment and it is unlikely that participating in the research affected their practice.

\section{Results}

\section{Accuracy of respondents}

Respondents overestimated patients' performance. Ten patients scored less than $25 \%$ but, none of the relatives, one nurse and only four doctors gave estimates in the lowest quartile. The highest patient score was 188 , mean (SE) 103.80 (11.51), but nine relatives, six nurses and five doctors estimated that patients would achieve the maximum score of 200 , far exceeding patients' performances.

The figure shows that the estimates from doctors, nurses and relatives, as given by their mean scores, appear to be inaccurate and 
Figure Mean comprehension score (WAB) and 95\% confidence intervals of estimates by doctors, nurses, and relatives.

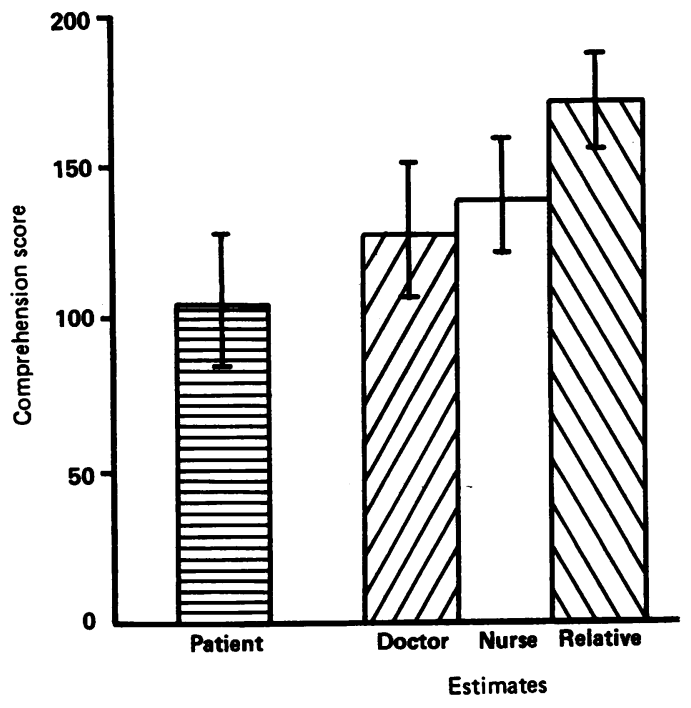

consistently greater than those of the patients.

All the estimated mean scores were significantly greater than the actual mean scores, relatives being least accurate in their estimates $(p<0.001)$ followed by nurses $(p=0.001)$ and then doctors $(p<0.05)$. However, despite their overall inaccuracy, it was still possible that respondents could discriminate those with severe difficulties from those with minor difficulties. Correlations between the patients' scores and the estimates by nurses, doctors and relatives were all significant $(r=0.59$, $\mathrm{p}<0.001 ; \mathrm{r}=0.55, \mathrm{p}<0.001$ and $\mathrm{r}=0.39$, $\mathrm{p}<0.05$ respectively).

\section{Agreement between respondents}

The estimates of comprehension ability from doctors, nurses and relatives were positively associated but the correlations were not significant (doctor-nurse $r=0.29$; doctor-relative $r=0.28$; nurse-relative $r=0.21$ ). This would appear to indicate that there was a lack of agreement not only between the health professionals and the relatives but also between the health professionals themselves.

\section{Discussion}

This study not only demonstrates the extent to which doctors, nurses and relatives underestimate the receptive disability of stroke patients, but also the lack of agreement between health professionals. It is clear that the WAB, as estimated by doctors, nurses and relatives in this study, would not give a valid measure of aphasia. The study may have omitted patients where the health professionals have failed to identify a language problem.

The overestimation of the aphasic patient's language abilities shown here by relatives has also been documented in several previous studies. Reasons for this positive bias may be lack of knowledge that this is a possible consequence of stroke, or emotional support for the relative that had become disabled and the view that the loss of comprehension was a stigma. It is unlikely that these reasons could also explain the overestimation made by the doctors and nurses in this study.

The experience of observing stroke patients is not new to doctors and nurses, and yet they overestimate the patient's receptive abilities. It is possible that respondents overestimate patients' comprehension abilities because they make no allowance for their own non-verbal cues as the means of communication with the patient.

The data suggest that the health professionals will frequently attempt to communicate with patients at a higher level than the patients can understand, thus exacerbating the normal problems of doctor-patient communication. This problem might be overcome by doctors and nurses using a brief standardised test validated for this purpose.

We thank Lena Rustin, Diana Moir, Wendon Dobbs and other members of the Speech Therapy Department, and Narinder Kapur and Noel Olsen for their contributions to the planning and execution of this study. We are grateful to all the patients, relatives, doctors and nurses who participated.

1 Wade DT, Langton Hewer R, David RM, Enderby PM. Aphasia after stroke: natural history and associated deficits. J Neurol Neurosurg Psychiatry 1986;49:11-16.

2 Johnston M, Bromley I, Boothroyd-Brooks M, Dobbs W, Ilson A, Ridout $\mathrm{K}$. Behavioural assessments of physically disabled patients: agreement between rehabilitation therapists and nurses. Int $J$ Rehabil Res 1987;10 (Supp 5):205-13

3 Flowers CR, Beukelman DR, Bottorf LE, Kelley RA. Family members' predictions of aphasic test performance. Aphasia-Apraxia-Agnosia 1979;162:18-25.

4 Helmick JW, Watamori TS, Palmer JM. Spouses' understanding of the communication disabilities of aphasic standing of the communication disabilities

5 Linebaugh CW, Young-Charles HY. The counseling needs of the families of aphasic patients. In: Brookshire RH, ed. Proc Clinical Aphasiology Conference, Minneapolis, MN: Proc Clin 1978.

6 Kertesz A, Poole E. The aphasia quotient: the taxonomic approach to measurement of aphasic disability. Canadian Journal of Neurological Sciences 1974;1:7-16.

7 Shewan CM, Kertesz A. Reliability and validity characteristics of the Western Aphasia battery (WAB). Journal of Speech and Hear Disord 1980;45:308-24. 